\title{
Diagnostic accuracy of dynamic CZT-SPECT in coronary artery disease. A systematic review and meta-analysis
}

\author{
Mariska Panjer, MD, ${ }^{\mathrm{a}}$ Magdalena Dobrolinska, MD, ${ }^{\mathrm{a}, \mathrm{b}}$ Nils R. L. Wagenaar, \\ $M D,{ }^{c, d}$ and Riemer H. J. A. Slart, MD, PhD ${ }^{\mathrm{a}, \mathrm{c}}$ \\ a Department of Nuclear Medicine and Molecular Imaging, Medical Imaging Center, University \\ Medical Center Groningen, University of Groningen, Groningen, The Netherlands \\ b Department of Cardiology and Structural Heart Diseases, Medical University of Silesia in \\ Katowice, Katowice, Poland \\ c Department of Biomedical Photonic Imaging, Faculty of Science and Technology, University of \\ Twente, Enschede, The Netherlands \\ d Department of Nuclear Medicine, Ziekenhuis Groep Twente, Hengelo, The Netherlands
}

Received Mar 31, 2021; accepted Jun 17, 2021

doi: $10.1007 / \mathrm{s} 12350-021-02721-8$

Background. With the appearance of cadmium-zinc-telluride (CZT) cameras, dynamic myocardial perfusion imaging (MPI) has been introduced, but comparable data to other MPI modalities, such as quantitative coronary angiography (CAG) with fractional flow reserve (FFR) and positron emission tomography (PET), are lacking. This study aimed to evaluate the diagnostic accuracy of dynamic CZT single-photon emission tomography (SPECT) in coronary artery disease compared to quantitative CAG, FFR, and PET as reference.

Materials and Methods. Different databases were screened for eligible citations performing dynamic CZT-SPECT against CAG, FFR, or PET. PubMed, OvidSP (Medline), Web of Science, the Cochrane Library, and EMBASE were searched on the 5th of July 2020. Studies had to meet the following pre-established inclusion criteria: randomized controlled trials, retrospective trails or observational studies relevant for the diagnosis of coronary artery disease, and performing CZT-SPECT and within half a year the methodological references. Studies which considered coronary stenosis between $50 \%$ and $70 \%$ as significant based only on CAG were excluded. Data extracted were sensitivity, specificity, likelihood ratios, and diagnostic odds ratios. Quality was assessed with QUADAS-2 and statistical analysis was performed using a bivariate model.

Results. Based on our criteria, a total of 9 studies containing 421 patients were included. For the assessment of CZT-SPECT, the diagnostic value pooled analysis with a bivariate model was calculated and yielded a sensitivity of 0.79 (\% CI 0.73 to 0.85$)$ and a specificity of $0.85(95 \%$ CI 0.74 to 0.92). Diagnostic odds ratio (DOR) was $17.82(95 \%$ CI 8.80 to $36.08, P<0.001)$. Positive likelihood ratio (PLR) and negative likelihood ratio (NLR) were 3.86 (95\% CI 2.76 to 5.38, $P<0.001)$ and $0.21(95 \%$ CI 0.13 to $0.33, P<0.001)$, respectively.

Supplementary Information The online version contains supplementary material available at https://doi.org/10.1007/s12350-02102721-8.

The authors of this article have provided a PowerPoint file, available for download at SpringerLink, which summarises the contents of the paper and is free for re-use at meetings and presentations. Search for the article DOI on SpringerLink.com.

Funding This study received no funding of any kind.

Reprint requests: Riemer H. J. A. Slart, MD, PhD, Department of Nuclear Medicine and Molecular Imaging, Medical Imaging Center,
University Medical Center Groningen, University of Groningen, Hanzeplein 1, 9700 RB Groningen, The Netherlands; r.h.j.a.slart@umcg.nl 1071-3581/\$34.00 Copyright (c) 2021 The Author(s) 
Conclusion. Based on the results of the current systematic review and meta-analysis, dynamic CZT-SPECT MPI demonstrated a good sensitivity and specificity to diagnose CAD as compared to the gold standards. However, due to the heterogeneity of the methodologies between the CZT-SPECT MPI studies and the relatively small number of included studies, it warrants further well-defined study protocols. (J Nucl Cardiol 2022;29:1686-97.)

Key Words: CZT-SPECT • Dynamic flow $\cdot$ CAD • Accuracy $\cdot$ Systematic review $\cdot$ Metaanalysis

See related editorial, pp. 1698-1701

\section{INTRODUCTION}

Non-invasive imaging which assesses myocardial perfusion (MPI) plays an important role in the diagnosis of coronary artery disease (CAD). ${ }^{1}$ It provides information about myocardial function which is superior to anatomical assessment alone., ${ }^{2,3}$ MPI can be assessed by positron emission tomography (PET), single-photon emission computed tomography (SPECT), magnetic resonance imaging (MRI), or computed tomography (CT).

For years, invasive coronary angiography (CAG) has been considered a gold standard in the CAD diagnosis. However, a primary concern of this method is the fact that it provides only anatomical assessment. Over the last decade, invasively measured fractional flow reserve (FFR) became a reference for the assessment of hemodynamic significance of coronary lesion and it is a standard of care in patients with angiographically assessed stenosis $>50 \%{ }^{1,3}$ Notwithstanding the clinical value of CAG and FFR, the invasive character, risk of radiation, and injection of contrast are the main limitation in daily practice.

Within abovementioned noninvasive methods, PET is considered a gold standard. However, due to expensive tracer production and high costs, it is mainly limited to major academic centers. In contrast to PET, SPECT is less expensive, wider, and more easily available. Traditionally, SPECT MPI evaluates defects in myocardial perfusion through a semi-quantitative 5-point analysis. It provides only relative assessment of myocardial perfusion which is inferior to quantitative analysis. Furthermore, its diagnostic accuracy is also limited by the low resolution and artifacts. ${ }^{4}$ However, the recently introduced CZT camera overcomes these technical limitations. Due to higher resolution and shorter acquisition time, it not only improves the overall SPECT performance but also decreases radiation dose..$^{5-7}$ Importantly, CZT cameras enable the quantification of myocardial blood flow (MBF) and myocardial flow reserve (MFR) which provides more accurate assessment of multivessel, left main, and microvascular disease than visual assessment or semi-quantitative perfusion evaluation. ${ }^{8}$ Therefore, we decided to focus on dynamic scans acquired with CZT-SPECT, based on which the quantitative, and thereby more precise, analysis of blood flow is available.
Recent trends in CZT-SPECT clinical application have led to the proliferation of studies investigating to estimate its diagnostic accuracy. However, the status of the comparison between dynamic CZT-SPECT and clinical diagnostic standards in functional assessment of CAD diagnosis is unclear so far. Until now, CZTSPECT was mostly compared to CAG, which can only assess the anatomical significance of the stenosis. Therefore, the primary aim of this systematic review and meta-analysis was to evaluate current evidence on the diagnostic accuracy of dynamic CZT-SPECT compared to gold standards of functional CAD assessment.

\section{MATERIALS AND METHODS}

\section{Data Source and Study Selection}

This systematic review and meta-analysis was performed according to PRISMA-DTA statement. ${ }^{1}$ Approval of Bioethical Committee was not necessary since it was obtained before publication of each included study. In order to identify suitable studies, the following databases were searched: PubMed, OvidSP (Medline), Web of Science, the Cochrane Library, and EMBASE. The MESH search strategy included the following key words: coronary artery disease or CAD or myocardial blood flow or myocardial ischemia and dynamic SPECT or cadmium zinc and telluride or CZT or D-SPECT and coronary angiography or CAG or fractional flow reserve or FFR or positron emission tomography or PET or coronary computed tomography angiography or CCTA or MRI or magnetic resonance imaging. Studies published up to the 5th of July 2020 were included in our analyses. We included all studies which reported to perform dynamic CZT-SPECT and within half a year the methodological references coronary angiography with or without FFR, positron emission tomography, magnetic resonance, or coronary computed tomography angiography. Studies which considered coronary stenosis between $50 \%$ and $70 \%$ as significant based only on CAG without FFR were excluded from this systematic review. The studies also required sufficient data to extract true positives, true negatives, false positives, and false negatives. The exclusion criteria were as follows: papers written in non-English, non-human trials, small patient numbers $(n<10)$, no full-text available, reviews, 
meta-analysis, editorials and case studies, inclusion of data duplicated in other studies. Screening was performed individually and independently by two reviewers; then, a consensus procedure followed any remaining inconsistencies were resolved by a third reviewer.

\section{Data Extraction and Quality Assessment}

Data extracted contained first author, publication year, study type, patient number, index test (dynamic CZT-SPECT), acquisition protocol, comparator, type of radiotracer, true positives, true negatives, false positives, false negatives, type of coronary artery disease and patient characteristics such as age, sex, diabetes mellitus, body mass index, obesity, hypertension, hyperlipidaemia, and percentage of prior CAD.

To assess study quality, Quality Assessment of Diagnostic Accuracy Studies (QUADAS-2) was used. Currently, QUADAS-2 is recommended to evaluate the quality of diagnostic studies. ${ }^{9}$ The criteria examined by QUADAS-2 are risk of bias (selection bias, index test bias, reference test bias, and flow timing bias) and applicability concerns according to each of bias risk. Selection bias risk was deemed high if patient exclusion was not explained and intermediate/unknown if the patient population consisted of patients with known CAD. The index test in this study is considered to be dynamic CZT-SPECT. Index test bias was deemed low if image assessment was blinded from other diagnostic tests, final diagnosis and if a threshold was pre-specified. If a study did not meet one of the criteria risks, risk of bias was deemed intermediate/unknown. All studies fulfilling only one criterion were deemed high risk. When reference standard analysis was blinded from dynamic CZT-SPECT outcomes, the final diagnosis bias risk was considered low. Flow timing bias was more difficult to judge since there is no established cut-off for the right timing between both tests. Therefore, no study is considered to have high-risk flow timing bias because only studies performing dynamic CZT-SPECT and the comparator within 6 months were included. The quality assessment was performed independently by two reviewers (Panjer and Dobrolinska), disagreement was solved by discussion or a third reviewer (Wagenaar) and is summarized in applicability concerns. The applicability concerns were considered low if studies met our inclusion criteria concerning the index test and reference standard. However, applicability concerns were deemed intermediate/unknown if studies had a patient population with $100 \%$ known CAD.

\section{Statistical Analysis}

Continuous variables were calculated as medians, and categorical variables were calculated as percentage. To assess the diagnostic accuracy of dynamic CZTSPECT, sensitivity, specificity, positive likelihood ratio (PLR), negative likelihood ratio (NLR), and diagnostic odds ratio (DOR) were used. A bivariate model was used with $95 \%$ confidence intervals (95\% CI). The bivariate model preserves the two-dimensional nature of the data throughout the analysis and takes into account the heterogeneity beyond chance between studies. In addition, a summary receiver-operating characteristic (SROC) curves were conducted to describe total diagnostic performance for both dynamic CZT-SPECT versus CAG, FFR and versus PET. The sROC curve is a plot of the true positive rate (sensitivity) as a function of the false positive rate (1-specificity). The heterogeneity was calculated as $I^{2}$. An $I^{2}$ higher than $50 \%$ was considered indicative of significant study heterogeneity. A $P$ value $<0.05$ was considered statistically significant. A meta-analysis was performed using Review Manager (RevMan, Version 5.3. Copenhagen: The Nordic Cochrane Centre, The Cochrane Collaboration, 2014), and heterogeneity was calculated with Open MetaAnalyst (BROWN School of Public Health, Providence, RI, USA).

\section{RESULTS}

\section{Search Results and Study Characteristics}

The results of study selection are visible in Fig. 1. After initial search, we identified 499 potentially relevant citations from which 15 studies were eligible for the primary study aim. No studies comparing CZTSPECT to CCTA or MRI were eligible for inclusion. From these full-text 15 reviewed articles, nine that fulfilled the inclusion criteria were ultimately included for the analysis.

We included mostly single-center, prospective cohort studies $(88.9 \%)$ which were published between 2015 and 2020. From nine studies, we encompassed altogether 421 patients. The sample size in the studies varied between 23 and 125 participants with median age of 66 years. Eight of the nine studies included patients with known CAD (89\%), five studies included patients $(55.5 \%)$ with previous myocardial infarction, and seven studies $(77.8 \%)$ included more men than women. Moreover, every study included patients diagnosed with diabetes mellitus (Table 1). 
Identification of studies via databases

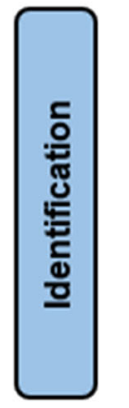

Records identified from:

PubMed $(n=101)$

Embase $(n=166)$

$\operatorname{OvidSP}(n=69)$

Web of Science $(n=163)$

Records removed before screening:

Duplicate records removed

$(n=199)$

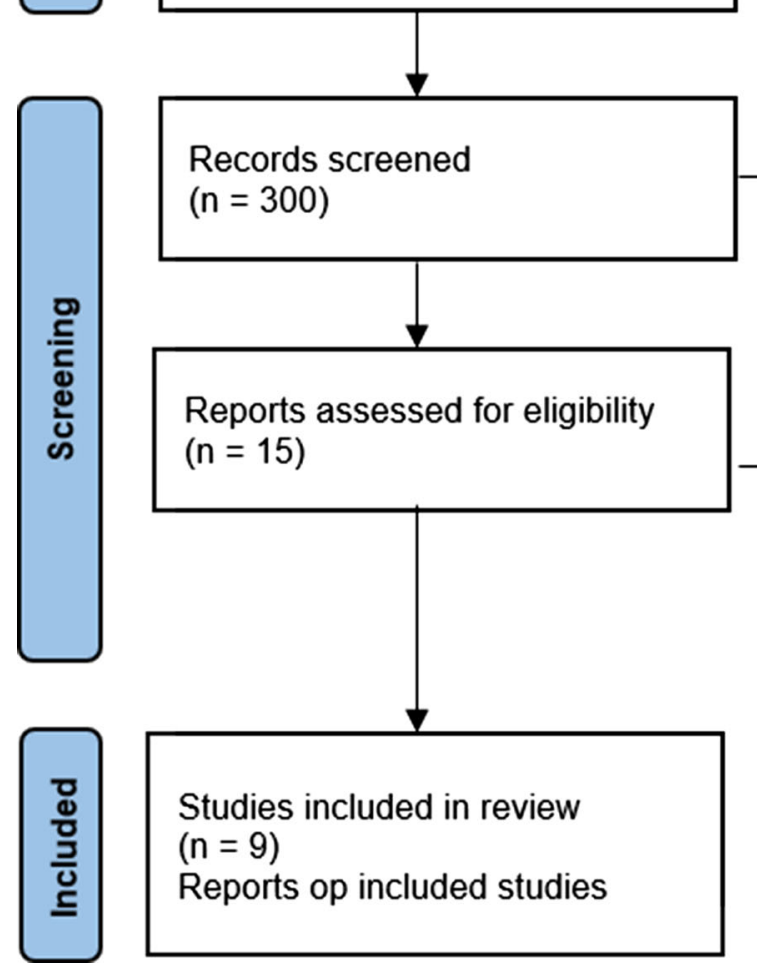

Records excluded

$(n=285)$

Figure 1. Flowchart of study selection process. Description of reviewing process for systematic review and meta-analysis.

Within included studies, seven used as a comparator FFR (77.8\%) and two used MPI-PET (22.2\%). The definition of stenosis based on FFR was slightly different. Three studies defined FFR $\leq 0.8(33.3 \%)$ and four FFR $<0.8(44.4 \%)$ as significant. According to PET studies, Agostini et al. performed ${ }^{15} \mathrm{O}$-water PET for MPR assessment and included an FFR defined abnormal if $\leq 0.8 .^{10}$

For the measurement of MPI, acquisition was performed with different types of CZT-SPECT cameras, six studies (66.7\%) used Discovery NM 530c (GE Healthcare, Chicago, IL, USA), one study (11.1\%) used Discovery NM/CT 570c (Alcyone technology, GE Healthcare, Haifa, Israel), and two studies (22.2\%) used D-SPECT (Spectrum Dynamics, Palo Alto, California).
Dual isotope administration was used in one study (11.1\%), two studies (22.2\%) used Thallium-201 as radiotracer, and six studies $(66.7 \%)$ used Technetium$99 \mathrm{~m}$ (Table 2). The mean or median radiation dose was reported in seven studies (77.8\%) and it was higher in Thallium-201 studies. Stress/rest protocol was used in half of the studies $(50 \%)$ and for the stress phase, the majority of the studies used adenosine (77.8\%). Importantly, different software were used for the MBF quantification and measurement of MFR, including inhouse software, with Corridor 4DM (INVIA, Ann Arbor, MI, USA) applied more frequently than others (Table 2). The method of diagnosing CAD was also different in the studies. Two studies ${ }^{10,11}(22.2 \%)$ performed a per-vessel analysis and the other seven 


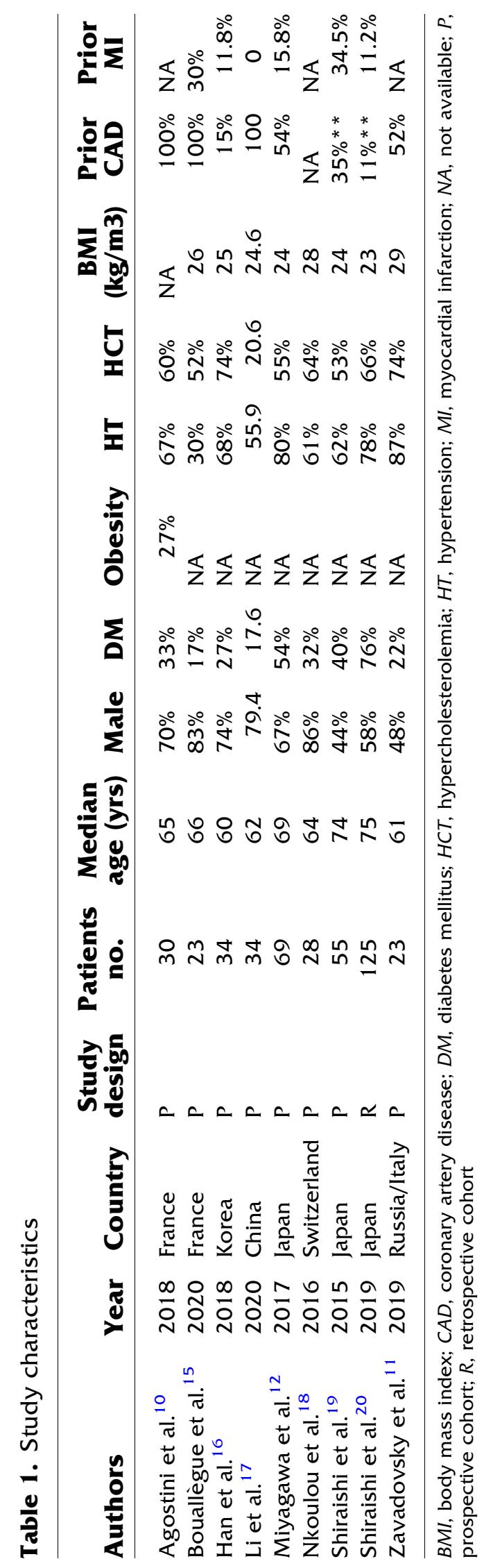




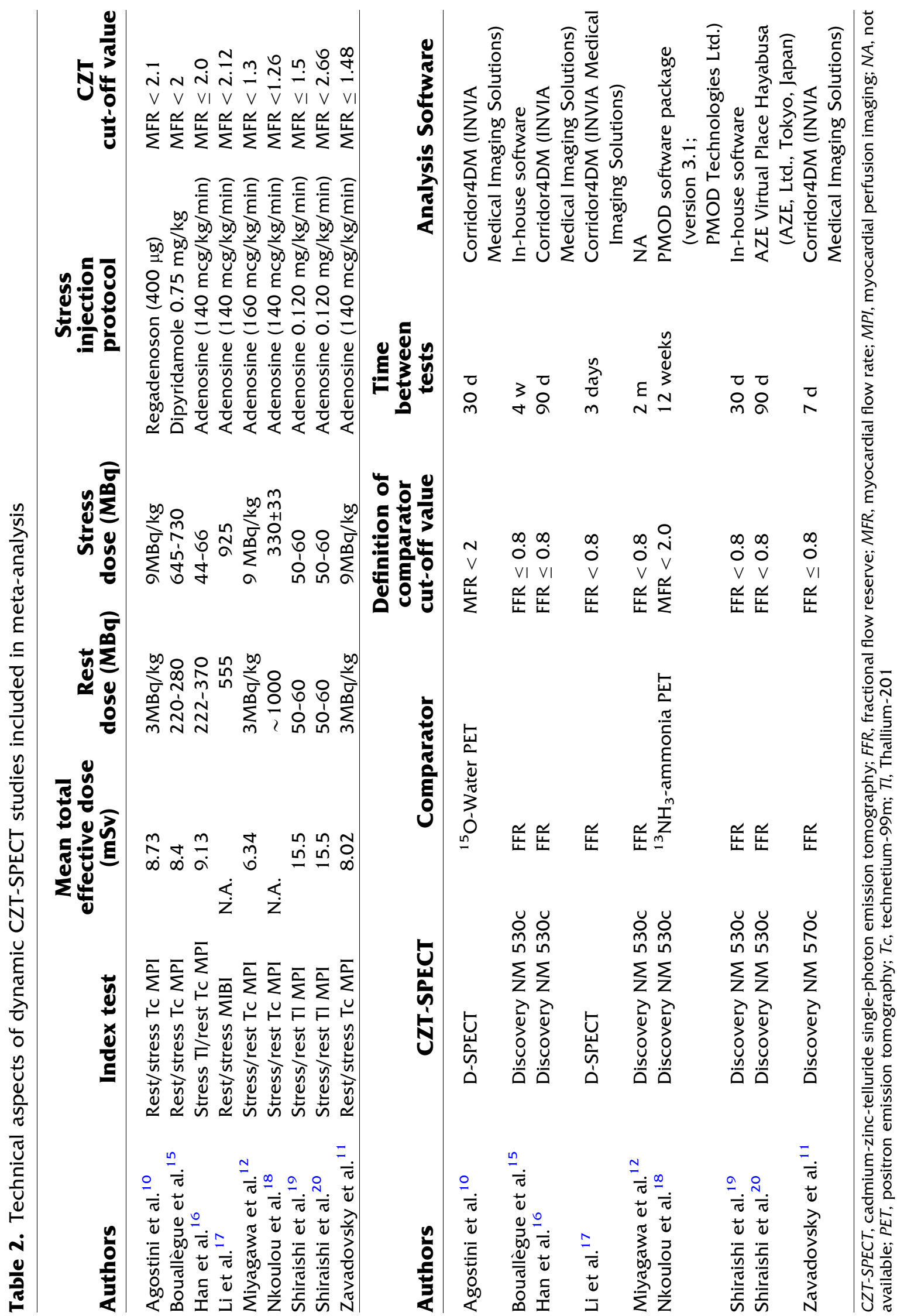


(77.8\%) performed a per-patient analysis. One study $(11.1 \%)$ diagnosed triple-vessel disease (TVD). ${ }^{12}$

\section{Methodological Quality of Included Studies}

Figure 2 summarizes the risk of bias and applicability concerns of the included studies. The applicability concerns were considered overall low. The domain which showed unclear risk of bias was 'patients' selection' due to inclusion of patients with previously known CAD. Only two studies $(22.2 \%)$ reported the reference standard to be assessed blinded, and all other studies did not specify blinding the data.

\section{Diagnostic Accuracy of Dynamic CZT-SPECT}

For the assessment of CZT-SPECT against both comparators PET and FFR, the diagnostic value pooled analysis with a bivariate model was calculated and yielded a sensitivity of $0.79(95 \%$ CI 0.73 to 0.85$)$ and a specificity of 0.85 (95\% CI 0.74 to 0.92) (Fig. 3, Table 3). An analysis of diagnostic accuracy is summarized on a SROC curve (Fig. 4). The subgroup that compared CZT-SPECT to FFR only $(n=7)$ demonstrated a similar sensitivity of $0.80(95 \% \mathrm{Cl} 0.73$ to 0.85$)$ but lower specificity of $0.78(\mathrm{Cl} 0.71$ to 0.83$)$ as compared to the whole group. Diagnostic odds ratio (DOR) was 17.82 (95\% CI 8.80 to $36.08, P<0.001$ ). Positive likelihood ratio (PLR) and negative likelihood ratio (NLR) were 3.86 (95\% CI 2.76 to $5.38, P<0.001$ ) and 0.21 (95\% CI 0.13 to $0.33, P<0.001$ ), respectively. Results are summarized in Figs. 5, 6, and 7. DOR, PLR, and NLR were calculated by a diagnostic random effects model. A Spearman's rank correlation test showed no evidence of a threshold effect, Spearman's correlation coefficient $=-0.3264(P=0.3914)$.

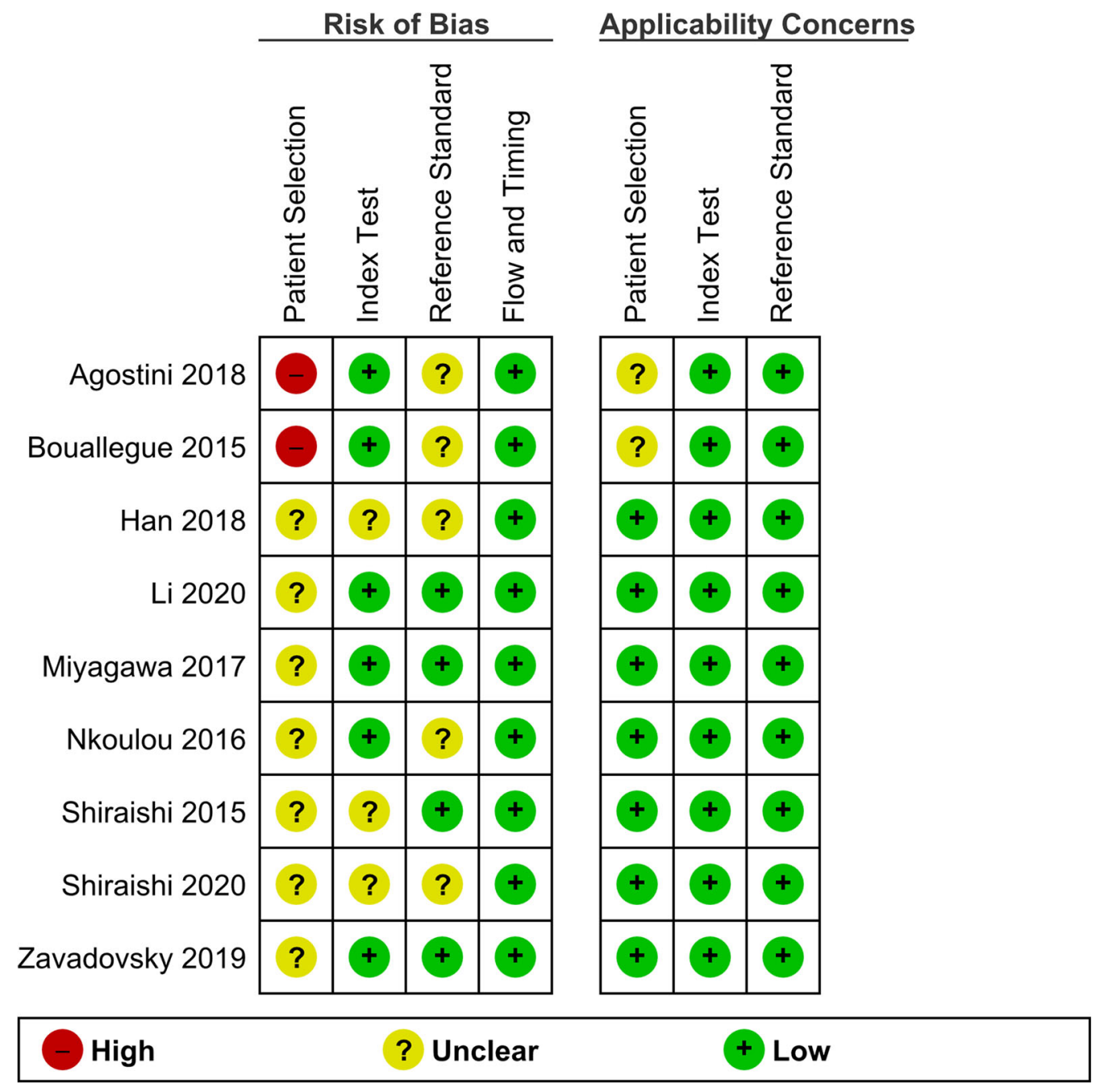

Figure 2. Quadas-2 risk of bias and applicability concerns summary. The summary of study inclusion criteria defined as low, unclear, and high, in terms of bias and applicability. 


$\begin{array}{lrrrrrrr}\text { Study } & \text { TP } & \text { FP } & \text { FN } & \text { TN } & \text { Sensitivity }(95 \% \mathrm{Cl}) & \text { Specificity }(95 \% \mathrm{Cl}) \\ \text { Agostini et al. 2018 } & 14 & 0 & 3 & 73 & 0.82[0.57,0.96] & 1.00[0.95,1.00] \\ \text { Bouallegue et al. 2015 } & 9 & 3 & 1 & 13 & 0.90[0.55,1.00] & 0.81[0.54,0.96] \\ \text { Han et al. 2018 } & 16 & 7 & 8 & 34 & 0.67[0.45,0.84] & 0.83[0.68,0.93] \\ \text { Li et al. 2020 } & 6 & 4 & 1 & 22 & 0.86[0.42,1.00] & 0.85[0.65,0.96] \\ \text { Miyagawa et al. 2017 } & 14 & 13 & 1 & 41 & 0.93[0.68,1.00] & 0.76[0.62,0.87] \\ \text { Nkoulou et al. 2016 } & 7 & 4 & 3 & 14 & 0.70[0.35,0.93] & 0.78[0.52,0.94] \\ \text { Shiraishi et al. 2015 } & 12 & 9 & 2 & 32 & 0.86[0.57,0.98] & 0.78[0.62,0.89] \\ \text { Shiraishi et al. 2020 } & 62 & 16 & 16 & 31 & 0.79[0.69,0.88] & 0.66[0.51,0.79] & \\ \text { Zavadovsky et al. 2019 } & 10 & 1 & 4 & 13 & 0.71[0.42,0.92] & 0.93[0.66,1.00]\end{array}$

Figure 3. Forest plot for sensitivity and specificity. $T P$, true positive; $F P$, false positive; $F N$, false negative; $T N$, true negative; $C I$, confidence interval.

\section{Heterogeneity Analysis}

Heterogeneity of this study was substantial for NLR, $I^{2}$ is $63 \%(P<0.006)$. For PLR and DOR, heterogeneity was moderate but not significant, 37\% $(P$ $<0.121)$ and $34 \%(P=0.149)$, respectively.

\section{DISCUSSION}

The high-sensitivity dedicated cardiac CZT-SPECT camera allows dynamic acquisition of tomographic images suitable for in vivo assessment of radiotracer kinetics and opens up a new era for myocardial flow and flow reserve measurement using SPECT imaging.

Our analysis was focused on the diagnostic accuracy of dynamic CZT-MPI SPECT. Based on this literature search and analysis, dynamic CZT-SPECT demonstrated a good sensitivity and specificity to diagnose coronary artery disease as compared to gold standards of functional and (quantitative) anatomical assessment.

The slightly reduced sensitivity of $79 \%$ in the current study might be explained by substantial discordance between FFR and MFR techniques. Notwithstanding the excellent diagnostic value of FFR, it quantifies the pressure gradient across the stenosis, but does not reflect microcirculation. As opposed to FFR, MFR reflects flow in epicardial arteries and microvasculature. ${ }^{15}$ It should be stressed that abnormal MFR with insignificant FFR indicates microvascular dysfunction or diffuse CAD. ${ }^{15,16}$ Therefore, FFR and MFR are not equivalent. ${ }^{15}$ Importantly, when CZT-SPECT MPI was compared to gold standard ${ }^{15} \mathrm{O}$-water PET, it gained an excellent accuracy. ${ }^{10}$ According to current guidelines, a diameter stenosis $>50 \%$ should be verified with FFR. Moreover, anatomical assessment does not reflect the MFR calculated with CZT-SPECT MPI. Therefore, we decided to include only studies which defined the comparator according to current gold standards, which includes functional assessment. This may explain a higher diagnostic odds ratio and specificity in the current meta-analysis as compared to CZT-SPECT MPI study of Nudi et al. ${ }^{13}$ This previous systematic review and metaanalysis on CZT-SPECT MPI has reported satisfactory sensitivity (0.84) and decreased specificity (0.69) of CZT-SPECT MPI. ${ }^{13}$ However, this decreased specificity might be due to the fact that MPI was compared to the \% diameter stenosis, which is only an anatomical measurement. Also, different comparator cut-off values of $\%$ diameter stenosis were used, including 50\% stenosis, and the included studies were mainly exist of static and not dynamic CZT-SPECT MPI scans. ${ }^{13}$

MPI is an important diagnostic tool for patients with known or suspected CAD. Therefore, a major effort was made to improve conventional SPECT MPI and replace it by CZT-SPECT camera.

The CZT-SPECT enables quantitative perfusion assessment due to its ultrafast signal processing. With dynamic cardiac acquisition, it is possible to detect the first-pass blood perfusion of tracer and its extraction into the myocardium allowing quantification of myocardial blood flow using dedicated compartment models. The CZT detector functions as a semiconductor with direct conversion of gamma radiation to an electric signal. This mechanism results in better spatial resolution and higher sensitivity, resulting in shorter acquisition time and/or lower radiation exposure. This offers superior performance advantages over sodium iodine detectors in Anger cameras. ${ }^{14}$

As a novelty, which enables to quantitatively measure myocardial perfusion by SPECT as MBF and MFR, it gained further interest. Taking both indices into 


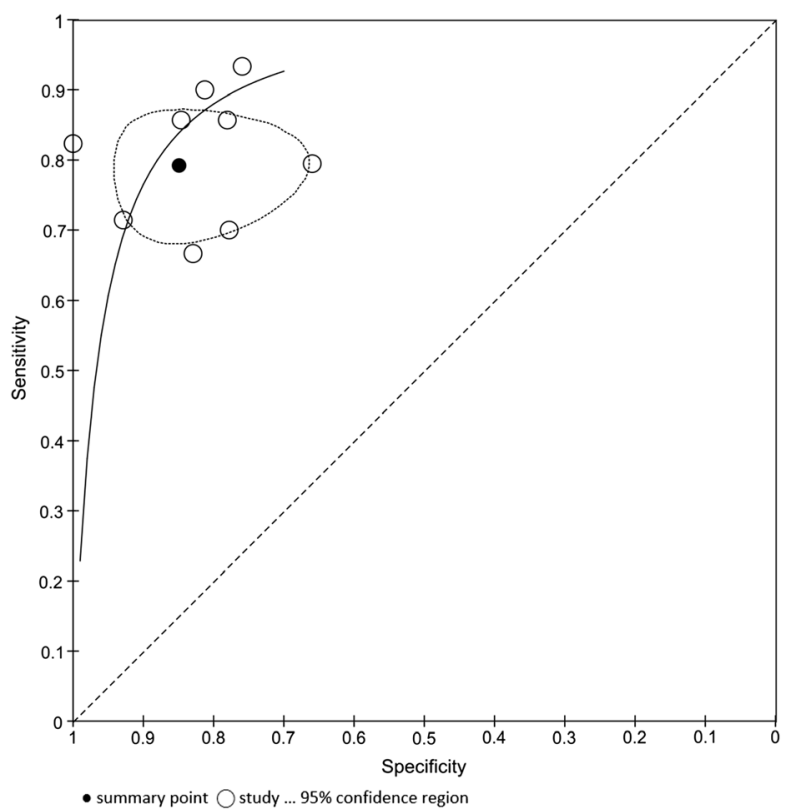

Figure 4. Summary Receiver Operating Curve (SROC) of included studies.

consideration, it was previously found that MFR yields higher diagnostic value as compared to MBF. ${ }^{10}$

\section{Study Limitations}

The main limitation of this systematic review and meta-analysis, additional to the typical limitations for this kind of analysis, is a relatively small number of included studies and patients and heterogeneity within comparators.

Heterogeneity between studies was substantial in terms of negative risk ratio and might be explained by effect modifiers. Firstly, different comparators were used. Moreover, there was also a diversity within study protocols, CZT cameras, radiotracers, dose administration, and software packages used for MPI CZT SPECT calculation. Furthermore, in each study, a different cutoff value for dynamic SPECT-MPI was set.

The present meta-analysis has mainly examined the CZT-MPI versus FFR. However, for further validation, CZT MPI should be compared to equivalent methods, including gold standard ${ }^{15} \mathrm{O}$-water PET or invasive coronary flow reserve (CFR) and index of microvascular resistance (IMR). 


\section{Studies}

Agostini et al. 2018

Bouallégue et al. 2015

Han et al. 2018

Li et al. 2020

Miyagawa et al. 2017

Nkoulou et al. 2016

Shiraishi et al. 2015

Shiraishi et al. 2020

Zavadovsky et al. 2019
Estimate (95\% C.I.)

$609.000(29.832,12432.294)$

$39.000 \quad(3.477,437.490)$

$9.714 \quad(2.998, \quad 31.475)$

$33.000 \quad(3.085, \quad 352.997)$

$44.154 \quad(5.287,368.775)$

8.167 (1.419, 47.016)

$21.333(4.017,113.286)$

7.508 (3.320, 16.981)

$32.500 \quad(3.127,337.813)$

$(\mathrm{TP} * \mathrm{TN}) /(\mathrm{FP} \star \mathrm{FN})$

Overall $\left(I^{\wedge} 2=33.55 \%, P=0.149\right)$

$17.820 \quad(8.802$ ，

36.077)

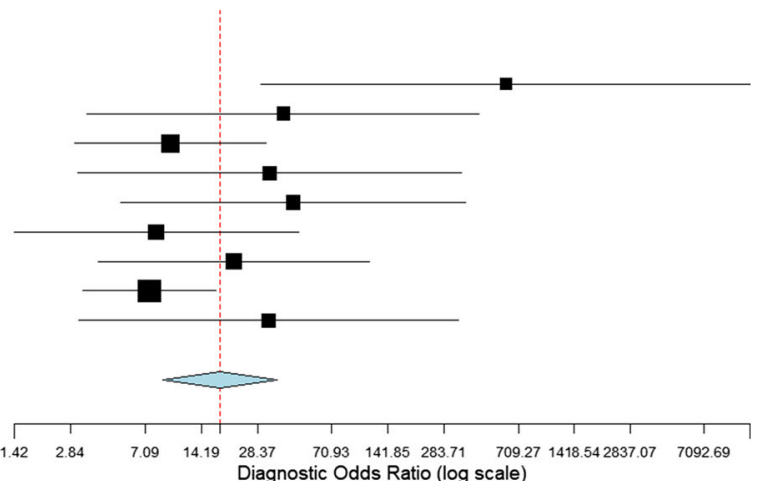

Figure 5. Forest plots for diagnostic odds ratio. C.I., confidence interval; TP, true positive; $T N$, true negative; $F P$, false positive; $F N$, false negative.

Studies

Agostini et al. 2018

Bouallégue et al. 2015

Han et al. 2018

Li et al. 2020

Miyagawa et al. 2017

Nkoulou et al. 2016

Shiraishi et al. 2015

Shiraishi et al. 2020

Zavadovsky et al. 2019

Positive Likelihood Ratio

Overall $\left(I^{\wedge} 2=37.24 \%, P=0.121\right)$

$\begin{array}{rrrr}\text { Estimate }(95 \% \text { C.I. }) & (\mathrm{TP} * \mathrm{Di}-) /(\mathrm{FP} * \mathrm{Di}+) \\ & & \\ 9.222 & (7.458, & 1905.929) & 1022 / 0 \\ 4.800(1.695, & 13.590) & 144 / 30 \\ 3.905(1.879, & 8.115) & 656 / 168 \\ 5.571 & (2.153, & 14.418) & 156 / 28 \\ 3.877 & (2.369, & 6.345) & 756 / 195 \\ 3.150(1.212, & 8.184) & 126 / 40 \\ 3.905(2.110, & 7.226) & 492 / 126 \\ 2.335(1.544, & 3.531) & 2914 / 1248 \\ 0.000 & (1.470, & 68.040) & 140 / 14\end{array}$

$3.855(2.762, \quad 5.379) \quad 6406 / 1849$

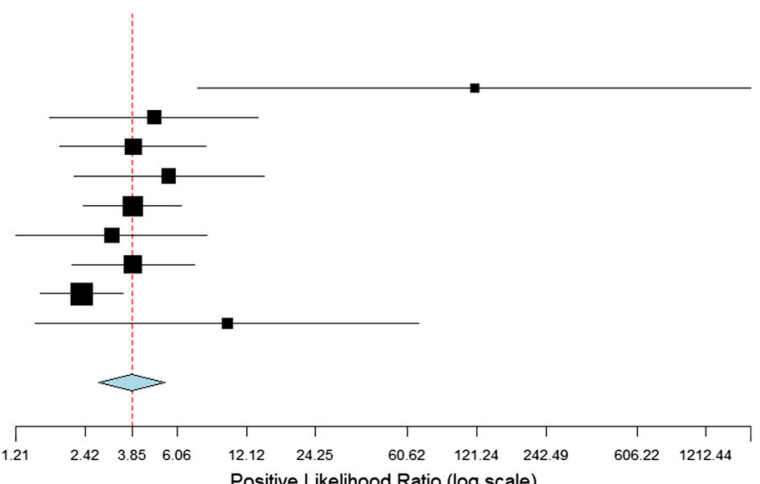

Figure 6. Forest plots for positive likelihood ratio. C.I., confidence interval; $T P$, true positive; $F P$, false positive; $D i-$, disease absent; $D i+$, disease present.

\section{Studies}

Agostini et al. 2018

Bouallégue et al. 2015

Han et al. 2018

Li et al. 2020

Miyagawa et al. 2017

Nkoulou et al. 2016

Shiraishi et al. 2015

Shiraishi et al. 2020

Zavadovsky et al. 2019

\section{Negative Likelihood Ratio}

$\begin{array}{lcc}\text { Estimate }(95 \% \mathrm{C} . \mathrm{I} .) & (\mathrm{FN} * \mathrm{Di}-) /(\mathrm{TN} * \mathrm{Di}+ \\ 0.196(0.012,3.130) & 219 / 1241 \\ 0.123(0.043,0.348) & 16 / 130 \\ 0.402(0.193,0.835) & 328 / 816 \\ 0.169(0.065,0.437) & 26 / 154 \\ 0.088(0.054,0.144) & 54 / 615 \\ 0.386(0.148,1.002) & 54 / 140 \\ 0.183(0.099,0.339) & 82 / 448 \\ 0.311(0.206,0.470) & 752 / 2418 \\ 0.308(0.045,2.094) & 56 / 182\end{array}$

Overall $\left(I^{\wedge} 2=62.98 \%, P=0.006\right) \quad 0.209(0.134,0.326)$

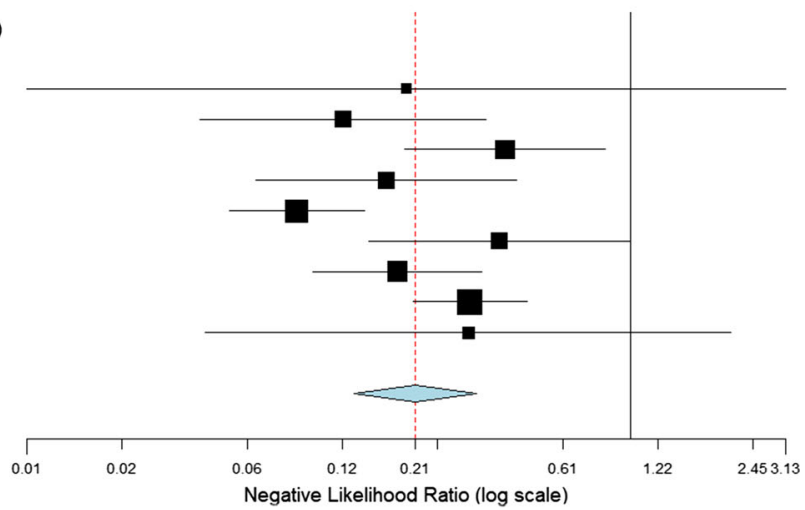

Figure 7. Forest plots for negative likelihood ratio. C.I., confidence interval; $F N$, false negative; $T N$, true negative; $D i-$, disease absent; $D i+$, disease present. 


\section{CONCLUSION}

Overall, dynamic CZT-SPECT MPI has a good diagnostic sensitivity and specificity for CAD diagnosis as compared to gold standards used in clinical practice. However, due to the heterogeneity of the methodologies between the CZT-SPECT MPI studies and the gold standards, and the relatively small number of included studies, it warrants further well-defined research protocols.

\section{NEW KNOWLEDGE GAINED}

The fact that CZT-SPECT MPI shows good diagnostic sensitivity and specificity for diagnosis of CAD indicates its value in everyday clinical practice.

\section{Disclosure}

The authors of this manuscript have no conflicts of interest to disclose.

\section{Open Access}

This article is licensed under a Creative Commons Attribution 4.0 International License, which permits use, sharing, adaptation, distribution and reproduction in any medium or format, as long as you give appropriate credit to the original author(s) and the source, provide a link to the Creative Commons licence, and indicate if changes were made. The images or other third party material in this article are included in the article's Creative Commons licence, unless indicated otherwise in a credit line to the material. If material is not included in the article's Creative Commons licence and your intended use is not permitted by statutory regulation or exceeds the permitted use, you will need to obtain permission directly from the copyright holder. To view a copy of this licence, visit http://creativecommons.org/licenses/by/4.0/.

\section{References}

1. Knuuti J, Wijns W, Achenbach S, Agewall S, Barbato E, Bax JJ, et al. 2019 ESC guidelines for the diagnosis and management of chronic coronary syndromes. Eur Heart J 2020;41:407-77.

2. Tonino PAL, Fearon WF, De Bruyne B, Oldroyd KG, Leesar MA, Ver Lee PN, et al. Angiographic versus functional severity of coronary artery stenoses in the FAME study. Fractional flow reserve versus angiography in multivessel evaluation. J Am Coll Cardiol 2010;55:2816-21. https://doi.org/10.1016/j.jacc.2009.11. 096.

3. Van Nunen LX, Zimmermann FM, Tonino PAL, Barbato E, Baumbach A, Engstrøm T, et al. Fractional flow reserve versus angiography for guidance of PCI in patients with multivessel coronary artery disease (FAME): 5-year follow-up of a randomised controlled trial. Lancet 2015;386:1853-60. https://doi. org/10.1016/S0140-6736(15)00057-4.

4. Burrell S, MacDonald A. Artifacts and pitfalls in myocardial perfusion imaging. J Nucl Med Technol 2006;34:193-211.

5. Bocher M, Blevis IM, Tsukerman L, Shrem Y, Kovalski G, Volokh L. A fast cardiac gamma camera with dynamic spect capabilities: Design, system validation and future potential. Eur J Nucl Med Mol Imaging 2010. https://doi.org/10.1007/s00259-0101488-z.

6. Esteves FP, Raggi P, Folks RD, Keidar Z, Wells Askew J, Rispler $\mathrm{S}$, et al. Novel solid-state-detector dedicated cardiac camera for fast myocardial perfusion imaging: Multicenter comparison with standard dual detector cameras. J Nucl Cardiol 2009. https://doi. org/10.1007/s12350-009-9137-2.

7. Erlandsson K, Kacperski K, Van Gramberg D, Hutton BF. Performance evaluation of D-SPECT: A novel SPECT system for nuclear cardiology. Phys Med Biol 2009. https://doi.org/10.1088/ 0031-9155/54/9/003.

8. Fiechter M, Ghadri JR, Gebhard C, Fuchs TA, Pazhenkottil AP, Nkoulou RN, et al. Diagnostic value of $13 \mathrm{~N}$-ammonia myocardial perfusion PET: Added value of myocardial flow reserve. J Nucl Med 2012. https://doi.org/10.2967/jnumed.111.101840.

9. Whiting PF, Rutjes AWS, Westwood ME, Mallett S, Deeks JJ, Reitsma JB, et al. QUADAS-2: A revised tool for the quality assessment of diagnostic accuracy studies. Ann Intern Med 2011;155:529-36. https://doi.org/10.7326/0003-4819-155-8-20111 0180-00009.

10. Agostini D, Roule V, Nganoa C, Roth N, Baavour R, Parienti JJ, et al. First validation of myocardial flow reserve assessed by dynamic 99mTc-sestamibi CZT-SPECT camera: Head to head comparison with 15O-water PET and fractional flow reserve in patients with suspected coronary artery disease. The WATERDAY study. Eur J Nucl Med Mol Imaging 2018. https://doi.org/10.1007/ s00259-018-3958-7.

11. Zavadovsky KV, Mochula AV, Boshchenko AA, Vrublevsky AV, Baev AE, Krylov AL, et al. Absolute myocardial blood flows derived by dynamic CZT scan vs invasive fractional flow reserve: Correlation and accuracy. J Nucl Cardiol 2019. https://doi.org/10. 1007/s12350-019-01678-z.

12. Miyagawa M, Nishiyama $Y$, Uetani T, Ogimoto A, Ikeda S, Ishimura $\mathrm{H}$, et al. Estimation of myocardial flow reserve utilizing an ultrafast cardiac SPECT: Comparison with coronary angiography, fractional flow reserve, and the SYNTAX score. Int J Cardiol 2017. https://doi.org/10.1016/j.ijcard.2017.06.012.

13. Nudi F, Iskandrian AE, Schillaci O, Peruzzi M, Frati G, BiondiZoccai G. Diagnostic accuracy of myocardial perfusion imaging with CZT technology: Systemic review and meta-analysis of comparison with invasive coronary angiography. JACC Cardiovasc Imaging 2017. https://doi.org/10.1016/j.jcmg.2016.10.023.

14. Kincl V, Drozdová A, Vašina J, Panovský R, Kamínek M. Cadmium-zinc-telluride SPECT scanners-new perspectives in nuclear cardiology. Cor Vasa 2015;57:e214-8.

15. Bouallègue FB, Roubille F, Lattuca B, Cung TT, Macia JC, Gervasoni $\mathrm{R}$, et al. SPECT myocardial perfusion reserve in patients with multivessel coronary disease: Correlation with angiographic findings and invasive fractional flow reserve measurements. J Nucl Med 2015. https://doi.org/10.2967/jnumed.114. 143164.

16. Han S, Kim YH, Ahn JM, Kang SJ, Oh JS, Shin E, et al. Feasibility of dynamic stress 201T1/rest 99mTc-tetrofosmin single photon emission computed tomography for quantification of 
myocardial perfusion reserve in patients with stable coronary artery disease. Eur J Nucl Med Mol Imaging 2018. https://doi.org/ 10.1007/s00259-018-4057-5.

17. Li C, Xu R, Yao K, Zhang J, Chen S, Pang L, et al. Functional significance of intermediate coronary stenosis in patients with single-vessel coronary artery disease: A comparison of dynamic SPECT coronary flow reserve with intracoronary pressure-derived fractional flow reserve (FFR). J Nucl Cardiol 2020. https://doi.org/ 10.1007/s12350-020-02293-z.

18. Nkoulou R, Fuchs TA, Pazhenkottil AP, Kuest SM, Ghadri JR, Stehli J, et al. Absolute myocardial blood flow and flow reserve assessed by gated SPECT with cadmium-zinc-telluride detectors using 99mTc-tetrofosmin: Head-to-head comparison with $13 \mathrm{~N}$ ammonia PET. J Nucl Med 2016. https://doi.org/10.2967/jnumed. 115.165498 .
19. Shiraishi S, Sakamoto F, Tsuda N, Yoshida M, Tomiguchi S, Utsunomiya D, et al. Prediction of left main or 3-vessel disease using myocardial perfusion reserve on dynamic thallium-201 single-photon emission computed tomography with a semiconductor gamma camera. Circ J 2015. https://doi.org/10.1253/circj. CJ-14-0932.

20. Shiraishi S, Tsuda N, Sakamoto F, Ogasawara K, Tomiguchi S, Tsujita K, et al. Clinical usefulness of quantification of myocardial blood flow and flow reserve using CZT-SPECT for detecting coronary artery disease in patients with normal stress perfusion imaging. J Cardiol 2020. https://doi.org/10.1016/j.jjcc.2019.09. 006 .

Publisher's Note Springer Nature remains neutral with regard to jurisdictional claims in published maps and institutional affiliations. 